\title{
Energetic Efficiency Biogas Plants Improvement by Integrated System: Biogas-Microalgae-Biofuels in Frame of Biorefinery Concept (Algal Biogas Concept Energy) ${ }^{+}$
}

\author{
Sanda Velea \\ National Institute for Research \& Development in Chemistry and Petrochemistry-ICECHIM Bucharest, \\ 202 Spl. Independentei, 060021 Bucharest, Romania; sanda.velea@icechim.ro \\ + Presented at the 15th International Symposium "Priorities of Chemistry for a Sustainable Development" \\ PRIOCHEM, Bucharest, Romania, 30th October-1st November 2019. \\ Published: 18 October 2019
}

Keywords: biogas; biomass; microalgae

Algal Biogas Concept Energy (2018-2021) is a national project financed by PN III Program, PN-III-P1-1.2-PCCDI-2017; Program 1-Development of national CD system; Subprogram 1.2-Institutional performance, complex projects developed in CDI consortia.

The complex project proposes the development and demonstration of innovative technologies to optimize the biogas plants by integrating the open ponds for microalgae cultivation using the digestate resulted from anaerobic digestion as a culture medium. The integral valorization of microalgae biomass consists in: obtaining of algal extracts (for co-digestion), lipid fraction (for biofuels), and spent biomass (co-digestion substrate); advanced separation and valorization of biogas by carbon dioxide conversion to bio-methane; biorefining of the solid digestate and lipid fractions obtained from algal biomass into biochar, fuel components, bitumen fluxes, bio-hydrogen, and liquefiable gaseous fractions. The results of this project will be: a demonstrated technology at TRL 6 level within the biogas-microalgae integrated and an experimental stand for the evaluation of bio-methane and liquefied gaseous hydrocarbons combustion efficiency and emission control. In order to achieve the main goals of the project, the project involves six partners.

The main objectives of the project:

1. Development of innovative technologies for the optimization of biogas plants functioning, by integration of open systems for mixotrophic cultivation of microalgae that use the liquid digestate resulted from anaerobic fermentation as a nutrient source and that produce algal extracts (fito-catalysts for co-fermentation process), lipid fraction (biofuel production), and spent algal biomass (as substrate for co-digestion);

2. Advanced valorization of biogas resulted by carbon dioxide conversion to bio-methane;

3. Processing, by bio-refining, of the solid digestate and lipid fractions from algal biomass for obtaining bio-coal, fuel components, bitumen fluxers, and liquefiable gaseous fractions;

4. Elaboration of an integrated demonstrative installation (experimental pilot), biogas-microalgae, for demonstration of optimized co-digestion technology functionality and for ensuring the instruction of research personnel from the consortium partner institutions; the experimental pilot installation will be placed at the headquarters of Partner 3-INCDCSZ, Brasov, andactivity conducted within Project 1 - coordinated by ICPE-CA;

5. Settingup an experimental stand for combustors, with gaseous fuels for characterization of the obtained liquefiable gaseous hydrocarbons; the stand will serve equally for research within the 
project, for research personnel training, and for analyses required for economic partners that want to characterize gaseous fuels, taking into account that there is no such laboratory existent at this moment in Romania; the experimental stand will be set up at Partner 5-UT, Iasi headquarters.

Acknowledgments: This work was supported by PN III Program, PN-III-P1-1.2-PCCDI-2017; Program 1 -Development of national CD system; Subprogram 1.2-Institutional performance, complex projects developed in CDI consortia, Contract 32PCCDI/2018.

(C) 2019 by the authors. Licensee MDPI, Basel, Switzerland. This article is an open access article distributed under the terms and conditions of the Creative Commons Attribution (CC BY) license (http://creativecommons.org/licenses/by/4.0/). 